\title{
Methylglyoxal mediates hyperalgesia in patients with painful diabetic neuropathy
}

The glycolytic metabolite methylglyoxal could be the key to explaining why some patients with diabetes develop hyperalgesia, according to research published in Nature Medicine. Peter Nawroth and his colleagues found that plasma levels of methylglyoxal were elevated in patients with painful diabetic neuropathy, and further experiments in cell culture and animal models indicated that the effects of this compound on nociception were being mediated through alterations in the voltage-gated sodium channel $\mathrm{Na}_{\mathrm{v}} 1.8$.

Previous studies had shown that methylglyoxal accumulation caused neuronal damage in the nematode worm Caenorhabditis elegans. Switching their attention to mammals, Nawroth's team set out to identify tissues that had low levels of the methylglyoxal-detoxifying enzyme glyoxalase, and might, therefore, be susceptible to accumulation of the metabolite. "Since this was neural tissue, the focus of our work went towards the issue of diabetic neuropathy," explains Nawroth.

The researchers recruited 20 patients with type 2 diabetes -10 with pain and 10 without pain-and 10 healthy controls. The patients with pain were found to have significantly raised plasma methylglyoxal levels compared with the individuals in the other two groups.

\section{4 ...systemic methylglyoxal treatment induced thermal and mechanical hyperalgesia... 77}

In wild-type mice, systemic methyglyoxal treatment induced thermal and mechanical hyperalgesia, accompanied by activation of brain regions known to be involved in pain processing. Such changes were not observed, however, when methylglyoxal was administered to $\mathrm{Na}_{\mathrm{v}} 1.8$ knockout mice. Cell culture experiments confirmed that methylglyoxal caused sensitization of sensory neurons, which was associated with post-translational modification of the $\mathrm{Na}_{\mathrm{v}} 1.8$ channel.

"What is new from our study is that methylglyoxal is formed intracellularly, and its plasma levels correlate with pain in a subset of diabetic patients," concludes Nawroth. The findings suggest that methylglyoxal could be a new target for the treatment of painful diabetic neuropathy.

Heather Wood

Original article Bierhaus, A. et al. Methylglyoxal modification of $\mathrm{Na}_{\mathrm{v}} 1.8$ facilitates nociceptive neuron firing and causes hyperalgesia in diabetic neuropathy. Nat. Med. doi:10.1038/nm.2750

Further reading Morcos, M. et al. Glyoxalase-1 prevents mitochondrial protein modification and enhances lifespan in Caenorhabditis elegans. Aging Cell 7, 260-269 (2008) 